\title{
Enhanced Electrical Transport in Carbon Nanotube Thin-Films through Defect Modulation
}

Jamie E. Rossi, ${ }^{1,2}$ Cory D. Cress, ${ }^{3}$ Sheila M. Goodman, ${ }^{1}$ Nathanael D. Cox, ${ }^{2,4}$ Ivan Puchades, ${ }^{1,2}$ Andrew R. Bucossi, ${ }^{2,4}$ Andrew Merrill, ${ }^{1,2}$ Brian J. Landi ${ }^{1,2^{*}}$

${ }^{1}$ Department of Chemical Engineering, Rochester Institute of Technology, Rochester, New York 14623, USA

${ }^{2}$ NanoPower Research Laboratory, Rochester Institute of Technology, Rochester, NY 14623, USA

${ }^{3}$ Electronics Science and Technology Division, United States Naval Research Laboratory, Washington D.C. 20375, USA

${ }^{4}$ Department of Microsystems Engineering, Rochester Institute of Technology, Rochester, New York 14623, USA

*Corresponding Author: Tel: (585) 475-4726, Email: brian.landi@ rit.edu (B.J. Landi) 
Single-wall carbon nanotubes (SWCNTs) were synthesized using laser vaporization of a graphite target containing $3 \mathrm{wt} \%$ of each $\mathrm{Ni}$ and Co catalysts. The as-produced soot was purified using a previously established procedure, ${ }^{1,2}$ followed by $\mathrm{HCl}$ treatment to remove residual metal catalyst. This process yields materials that are comparable to a 100\% pure SWCNT reference based on carbonaceous content from analysis of the optical absorbance spectrum (see Figure S1a), and TGA confirms < $1 \%$ metal catalyst content (see Figure S1b). SEM analysis of the SWCNT starting material qualitatively corroborates the sample purity, with little-to-no carbonaceous or metal catalyst impurities observed in the representative micrograph shown in Figure S1d. Likewise, Raman spectroscopy is known to be a good quantitative metric for assessing the quality of SWCNT materials, as the D/G Raman ratio provides information about the amount of disorder in a sample and scales proportionally with increasing defect content. ${ }^{3,4}$ The Raman spectra of the purified SWCNTs used in the current study (see Figure S1c) exhibits a $\mathrm{D} / \mathrm{G}$ ratio of 0.15 , which further demonstrates the relative quality of the SWCNT starting material. This purified SWCNT material is subsequently used in the fabrication of thin-film test structures for systematic ion irradiation and thermal annealing experiments.
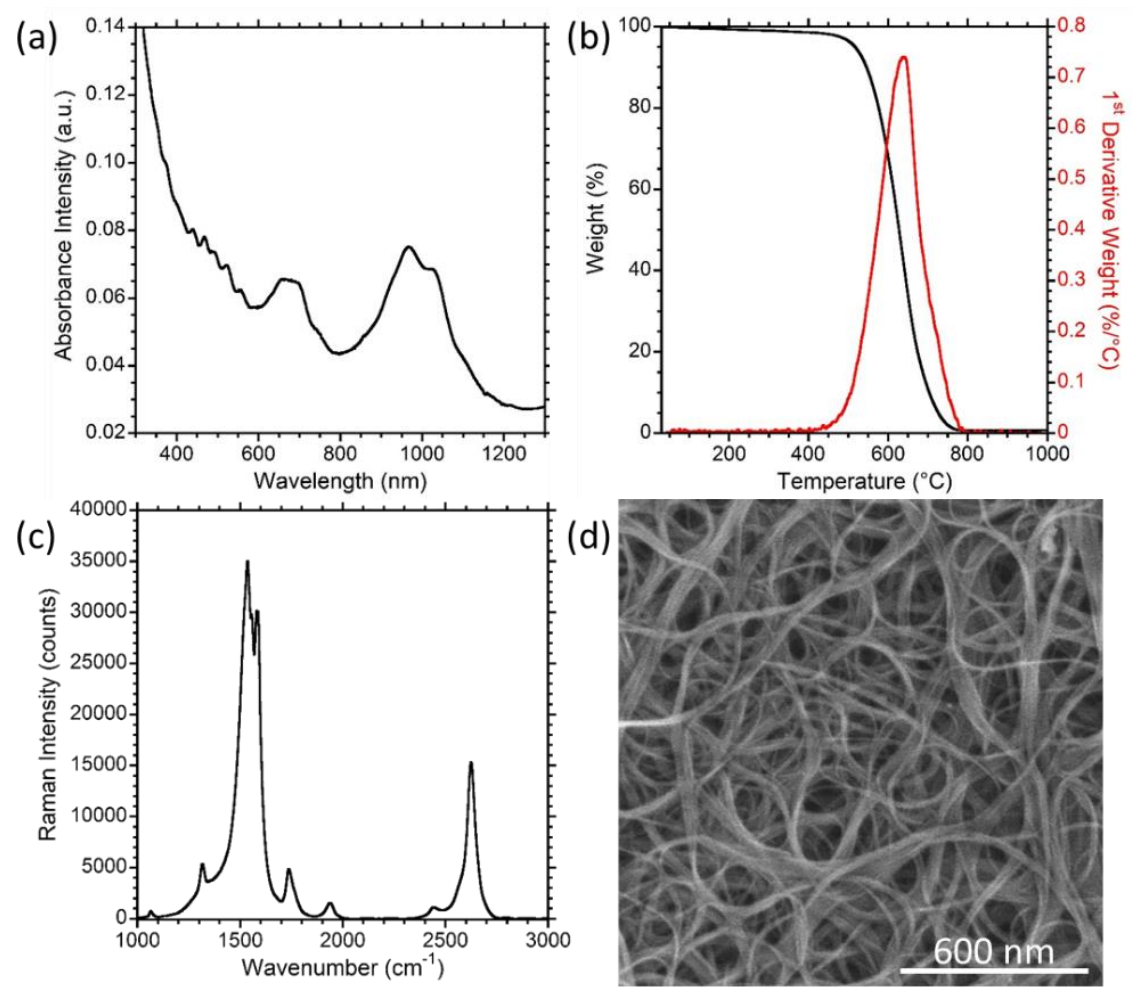

Figure S1. Purity of the SWCNT starting material was evaluated quantitatively through (a) optical absorbance spectroscopy, (b) thermogravimetric analysis, and (c) Raman spectroscopy, and qualitatively using (d) scanning electron microscopy. 
Five SWCNT thin-film samples were fabricated, transferred to polished quartz substrates, and purified using the optimized thermal oxidation (air, $2 \mathrm{~h}, 300^{\circ} \mathrm{C}$ ) and annealing $\left(\mathrm{H}_{2} / \mathrm{Ar}, 1 \mathrm{~h}\right.$, $1000^{\circ} \mathrm{C}$ ) treatments. The baseline optical absorbance and Raman spectra (see Figure S2a and Figure S2b, respectively) show that the intrinsic material properties are uniform between all five samples. Thermally evaporated $\mathrm{Cr} / \mathrm{Au}(10 / 200 \mathrm{~nm})$ contacts were deposited to aid in the electrical characterization of the final test structures, where the average sheet resistance $\left(R_{s}\right)$ was measured as $2.77 \mathrm{k} \Omega / \square \pm 1.04 \mathrm{k} \Omega / \square$ (see Figure S2c). After ion irradiation, and subsequent thermal annealing, changes in the SWCNT optical and electrical properties were related back to the as-purified condition measured for each sample.
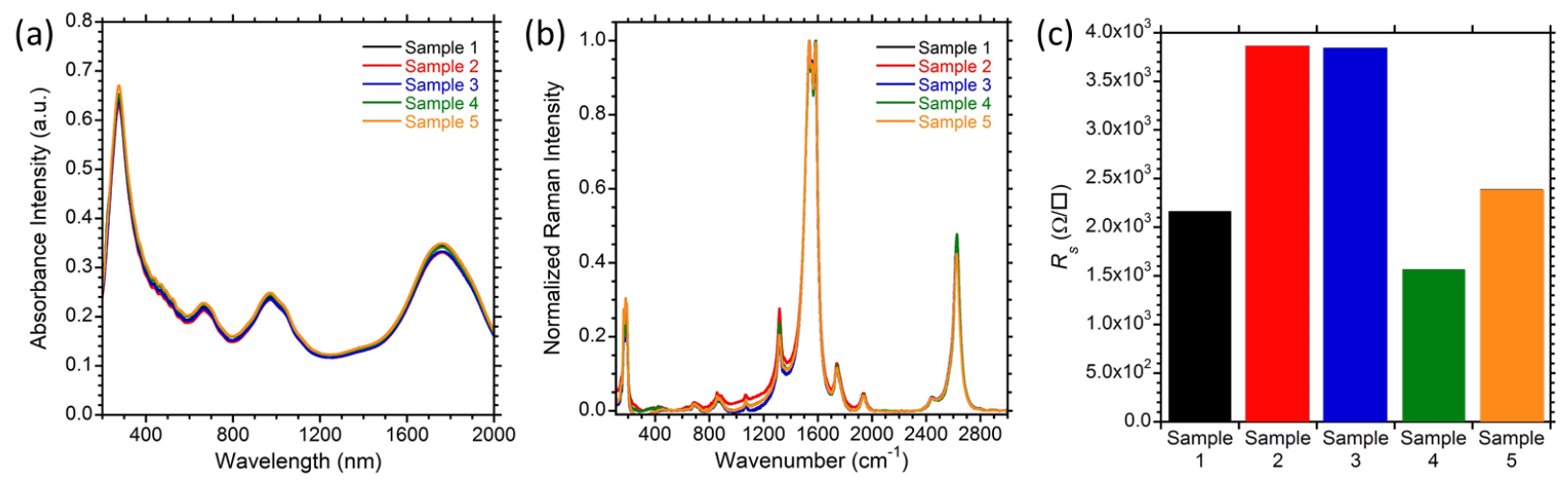

Figure S2. The five purified SWCNT thin-film test structures were characterized via (a) optical absorbance spectroscopy, (b) Raman spectroscopy, and (c) 4-point probe resistance measurements after thermal oxidation $\left(300^{\circ} \mathrm{C}\right.$, air, $\left.0.5 \mathrm{~h}\right)$ and thermal annealing $\left(1000^{\circ} \mathrm{C}\right.$, forming gas, $\left.1 \mathrm{~h}\right)$.

The thickness of the SWCNT films was characterized using a Veeco Wyko NT1100 Optical Profiling System with white light interferometry. Three line scans were measured over a representative portion of the sample (see Figure S3a). The thickness of the SWCNT film was determined by averaging a $0.2 \mathrm{~mm}$ distance from the film edge for each line scan and for the quartz substrate, which corresponds to an average film thickness of $27 \pm 8 \mathrm{~nm}$ (see Figure S3b).
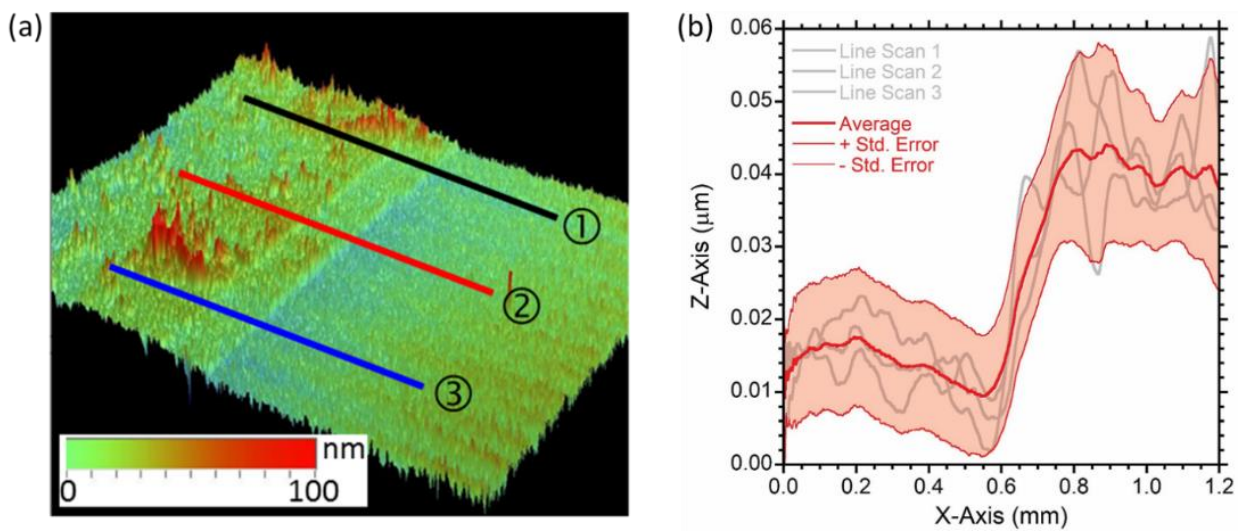

Figure S3. (a) The 3D step edge of a representative as-purified SWCNT thin-film on quartz was characterized using optical profilometry. (b) Three line scans were obtained, and used to determine the average thickness of the as-purified SWCNT test structure. 
Optical absorbance spectroscopy was used to characterize changes in the $E_{22}^{S}$ (see Figure $S 4$ ) and $E_{11}^{M}$ (see Figure S5) optical transitions after ion irradiation and thermal annealing. In both cases, the background subtracted SWCNT optical transitions are suppressed monotonically after ion irradiation with increasing fluence (red curves). Characterization of the samples after subsequent thermal annealing shows considerable recovery in both $E_{22}^{S}$ and $E_{11}^{M}$ (blue curves) at every level of defect concentration. After a low dose radiation exposure (i.e., $1 \times 10^{13}$ ions $/ \mathrm{cm}^{2}$ ) and thermal annealing, both $E_{22}^{S}$ and $E_{11}^{M}$ peaks show nearly $100 \%$ recovery in the optical transition relative to the as-purified condition. Even at high fluences annealing causes recovery in the optical transitions, though to varying degrees.
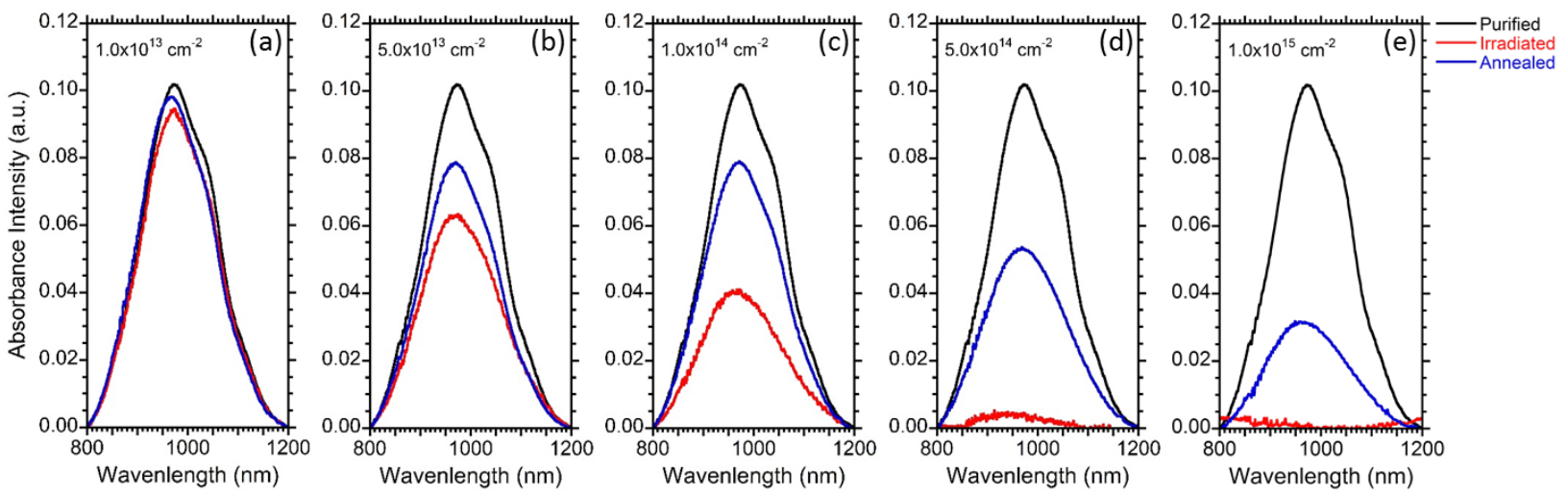

Figure S4. The background subtracted $E_{22}^{S}$ optical transition was analyzed for SWCNT thin-films after ion irradiation (red curves) with $150 \mathrm{keV}{ }^{11} \mathrm{~B}^{+}$to fluences of (a) $1 \times 10^{13}$ ions $/ \mathrm{cm}^{2}$, (b) $5 \times 10^{13}$ ions $/ \mathrm{cm}^{2}$, (c) $1 \times 10^{14}$ ions $/ \mathrm{cm}^{2}$, (d) $5 \times 10^{14}$ ions $/ \mathrm{cm}^{2}$, and (e) $1 \times 10^{15}$ ions $/ \mathrm{cm}^{2}$, compared to the as-purified control data (black curves). Also plotted is the $E_{22}^{S}$ optical transition after thermal annealing in forming gas at $1000^{\circ} \mathrm{C}$ for $1 \mathrm{~h}$ (blue curves).

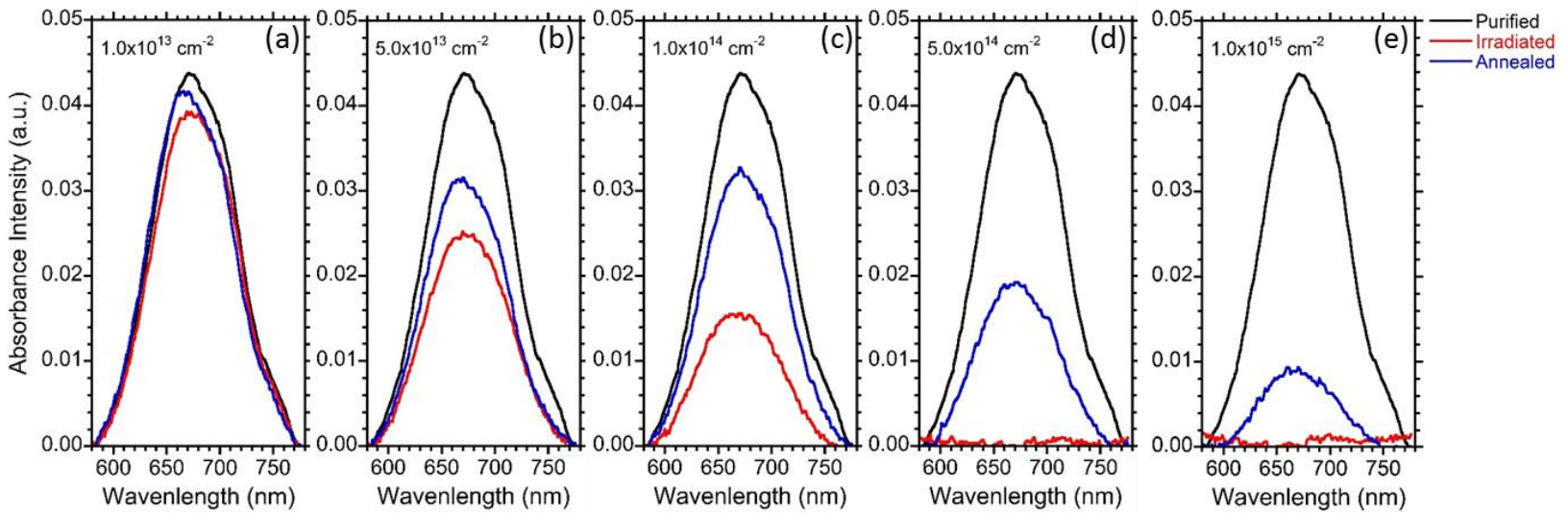

Figure S5. The background subtracted $E_{11}^{M}$ optical transition was analyzed for SWCNT thin-films after ion irradiation (red curves) with $150 \mathrm{keV}{ }^{11} \mathrm{~B}^{+}$to fluences of (a) $1 \times 10^{13}$ ions $/ \mathrm{cm}^{2}$, (b) $5 \times 10^{13}$ ions $/ \mathrm{cm}^{2}$, (c) $1 \times 10^{14}$ ions $/ \mathrm{cm}^{2}$, (d) $5 \times 10^{14}$ ions $/ \mathrm{cm}^{2}$, and (e) $1 \times 10^{15}$ ions $/ \mathrm{cm}^{2}$, compared to the as-purified control data (black curves). Also plotted is the $E_{11}^{M}$ optical transition after thermal annealing in forming gas at $1000^{\circ} \mathrm{C}$ for $1 \mathrm{~h}$ (blue curves). 
Analysis of the normalized $E_{22}^{S}$ (see Figure S6a) and $E_{11}^{M}$ (see Figure S6b) peaks demonstrate the monotonic decrease in peak intensity after ion irradiation with increasing fluence relative to the as-purified condition. Subsequent thermal annealing causes an increase in the relative peak intensity for both the $E_{22}^{S}$ and $E_{11}^{M}$, demonstrating recovery in the optical transitions.
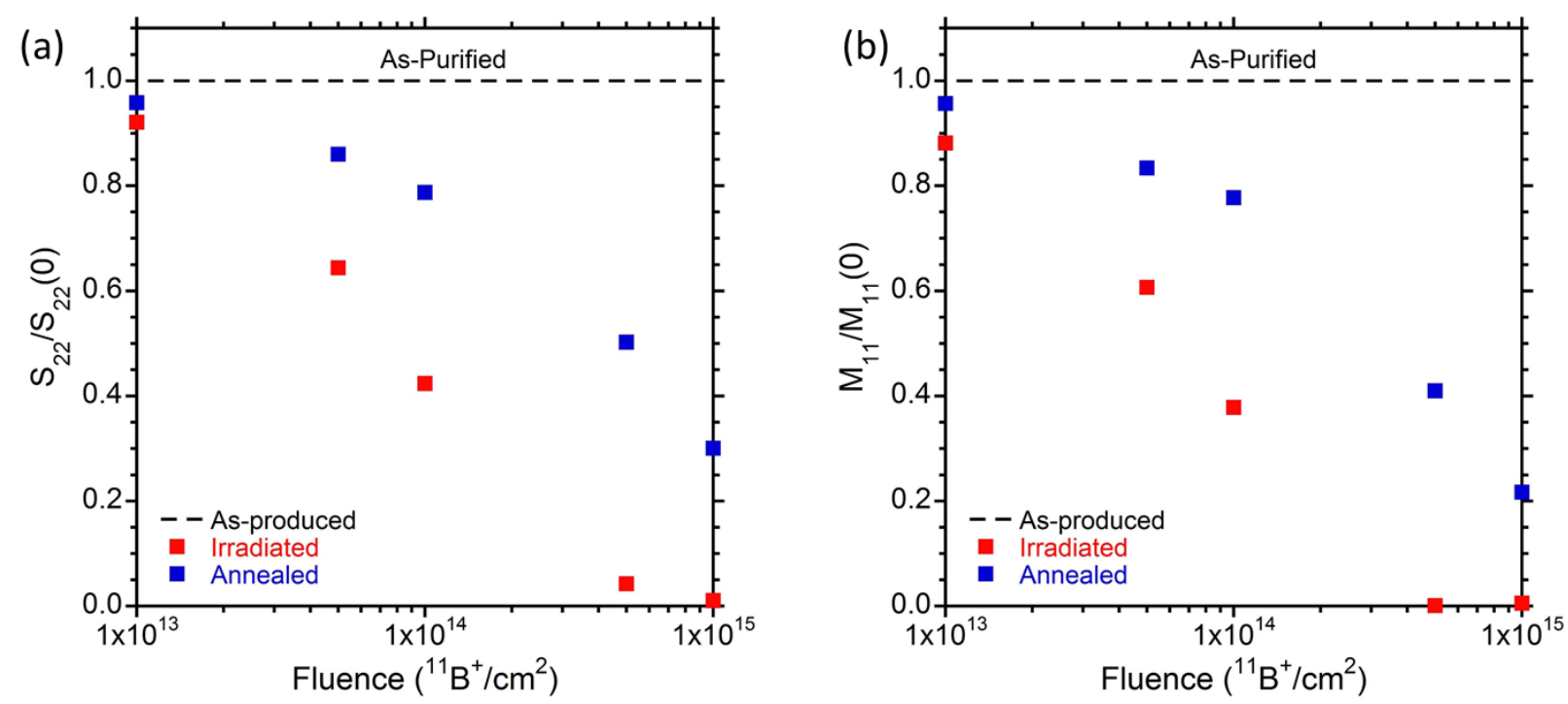

Figure S6. The relative peak maximum intensity of the (a) $E_{22}^{S}$ and (b) $E_{11}^{M}$ optical transition is plotted as a function of fluence before (red markers) and after (blue markers) thermal annealing. The data is normalized to the peak maximum intensity of the respective purified SWCNT control sample $\left(\mathrm{S}_{22}(0)\right.$ or $\left.\mathrm{M}_{11}(0)\right)$. 
Raman spectroscopy was used to assess changes in the SWCNT structure after radiation exposure and annealing by analyzing the $\mathrm{D}, \mathrm{G}$, and $\mathrm{G}^{\prime}$ bands (see Figure $\mathrm{S} 7$ ) and the radial breathing mode (RBM, see Figure S8). The Raman spectra of each irradiated and annealed SWCNT thin-film was normalized to the G peak maximum intensity of the respective as-purified control so that relative changes could be easily assessed. The red curves show a decrease in the $\mathrm{G}, \mathrm{G}^{\prime}$, and RBM peak intensities with increasing fluence, while the D peak initially increases. Following thermal annealing (blue curves), considerable recovery in the $G, G^{\prime}$, and RBM peak intensities is observed at all fluences examined. In particular, near complete recovery of the $G$, $\mathrm{G}^{\prime}$, and RBM peaks is observed after low dose radiation exposure (i.e., $\leq 5 \times 10^{13}$ ions $/ \mathrm{cm}^{2}$ ) and subsequent thermal annealing.

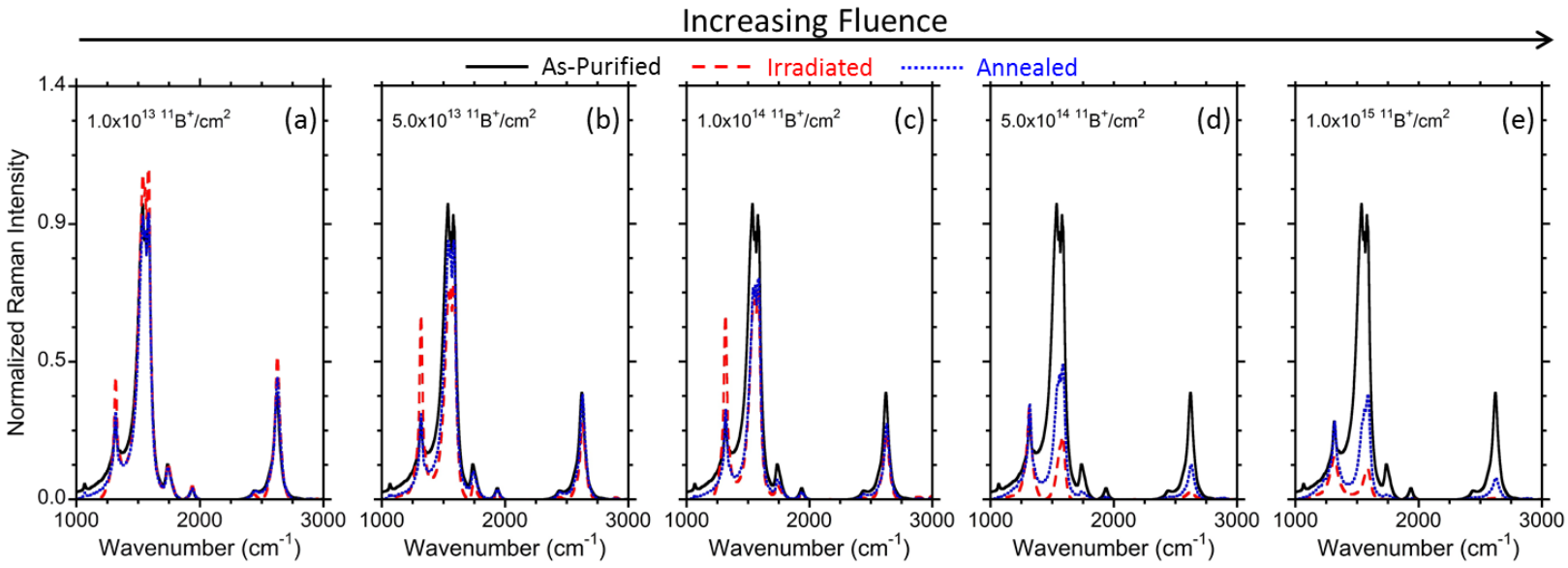

Figure S7. Analysis of the Raman D $\left(\sim 1320 \mathrm{~cm}^{-1}\right), \mathrm{G}\left(\sim 1540 \mathrm{~cm}^{-1}\right)$, and $\mathrm{G}^{\prime}\left(\sim 2620 \mathrm{~cm}^{-1}\right)$ peaks after ion irradiation of the SWCNT thin-films (red curves) with $150 \mathrm{keV}^{11} \mathrm{~B}^{+}$to fluences of (a) $1 \times 10^{13} \mathrm{ions} / \mathrm{cm}^{2}$, (b) $5 \times 10^{13} \mathrm{ions} / \mathrm{cm}^{2}$, (c) $1 \times 10^{14}$ ions $/ \mathrm{cm}^{2}$, (d) $5 \times 10^{14}$ ions $/ \mathrm{cm}^{2}$, and (e) $1 \times 10^{15}$ ions $/ \mathrm{cm}^{2}$. Also plotted is the purified control data (black curves) and annealed data (blue curves). All data is normalized to the $\mathrm{G}$ peak maximum intensity of the as-purified control sample.

Increasing Fluence

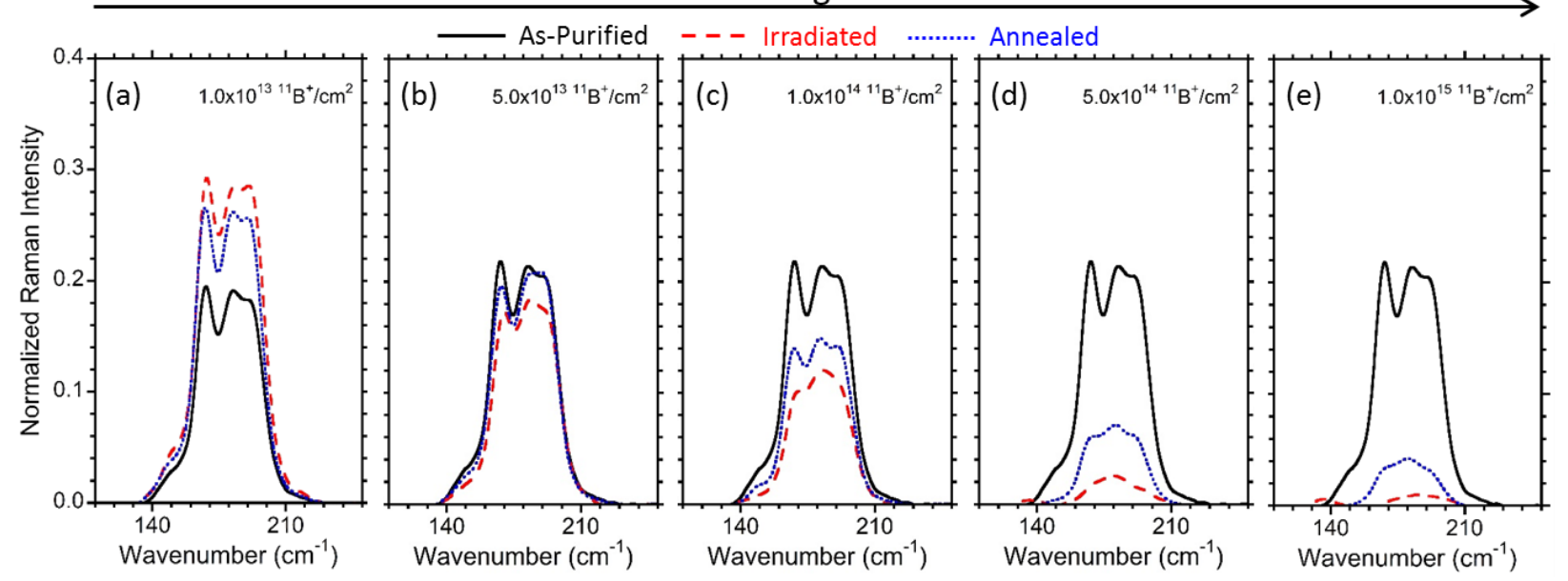

Figure S8. Analysis of the Raman radial breathing mode (RBM) peaks after ion irradiation of the SWCNT thinfilms (red curves) with $150 \mathrm{keV}{ }^{11} \mathrm{~B}^{+}$to fluences of (a) $1 \times 10^{13}$ ions $/ \mathrm{cm}^{2}$, (b) $5 \times 10^{13}$ ions $/ \mathrm{cm}^{2}$, (c) $1 \times 10^{14}$ ions $/ \mathrm{cm}^{2}$, (d) $5 \times 10^{14}$ ions $/ \mathrm{cm}^{2}$, and (e) $1 \times 10^{15}$ ions $/ \mathrm{cm}^{2}$. Also plotted is the purified control data (black curves) and annealed data (blue curves). All data is normalized to the G peak maximum intensity of the as-purified control sample. 
Analysis of the relative D/G Raman ratio allows for changes in the SWCNT structural properties and defect concentration to be easily assessed. Figure S9 (red markers) shows the change in D/G of the irradiated SWCNT thin-films relative to their as-purified condition, where a monotonic increase in $D / G$ as a function of fluence is observed. Figure S9 (blue markers) also shows recovery in the $\mathrm{D} / \mathrm{G}$ Raman response after subsequent thermal annealing at all fluences examined.

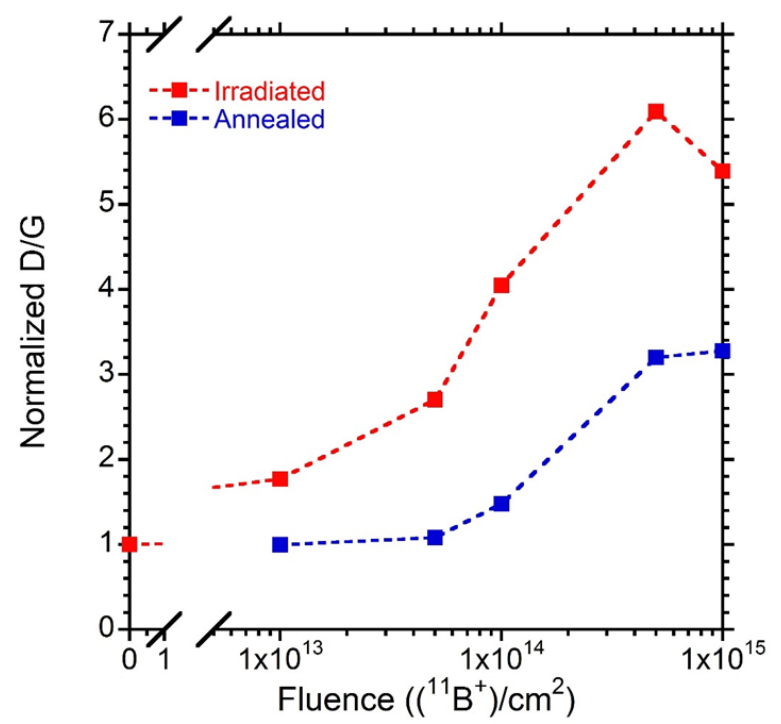

Figure S9. The relative D/G Raman ratio for SWCNT thin-films after ion irradiation with $150 \mathrm{keV}{ }^{11} \mathrm{~B}^{+}$(red markers), and after subsequent thermal annealing in $\mathrm{H}_{2} / \mathrm{Ar}(5 \% / 95 \% \mathrm{~mol} / \mathrm{mol})$ for $1 \mathrm{~h}$ at $1000^{\circ} \mathrm{C}$ (blue markers). All data is normalized to the D/G Raman ratio of the as-purified SWCNT control sample. 
A previously published electroless gold tagging method has been extended to the current work, ${ }^{5}$ which provides a means to spatially profile the density and distribution of defects in SWCNTs from ion irradiation, and characterize the recovery resulting from subsequent thermal annealing. The representative SEM micrograph in Figure S10a shows a SWCNT sample irradiated with 150 $\mathrm{keV}{ }^{11} \mathrm{~B}^{+}$to a fluence of $1 \times 10^{15}$ ions $/ \mathrm{cm}^{2}$ after Au-NP deposition (0.01 M KAuBr $\left.4(\mathrm{aq}), 30 \mathrm{sec}\right)$. There is a significant decrease in the Au-NP density when the sample is tagged after ion irradiation and subsequent thermal annealing (see Figure S10b). The decrease in Au-NP density correlates with the changes observed in the $\mathrm{D} / \mathrm{G}^{\prime}$ Raman ratio, following a similar trend as reported previously, and further corroborating recovery in the SWCNT physical properties through defect healing after thermal annealing.
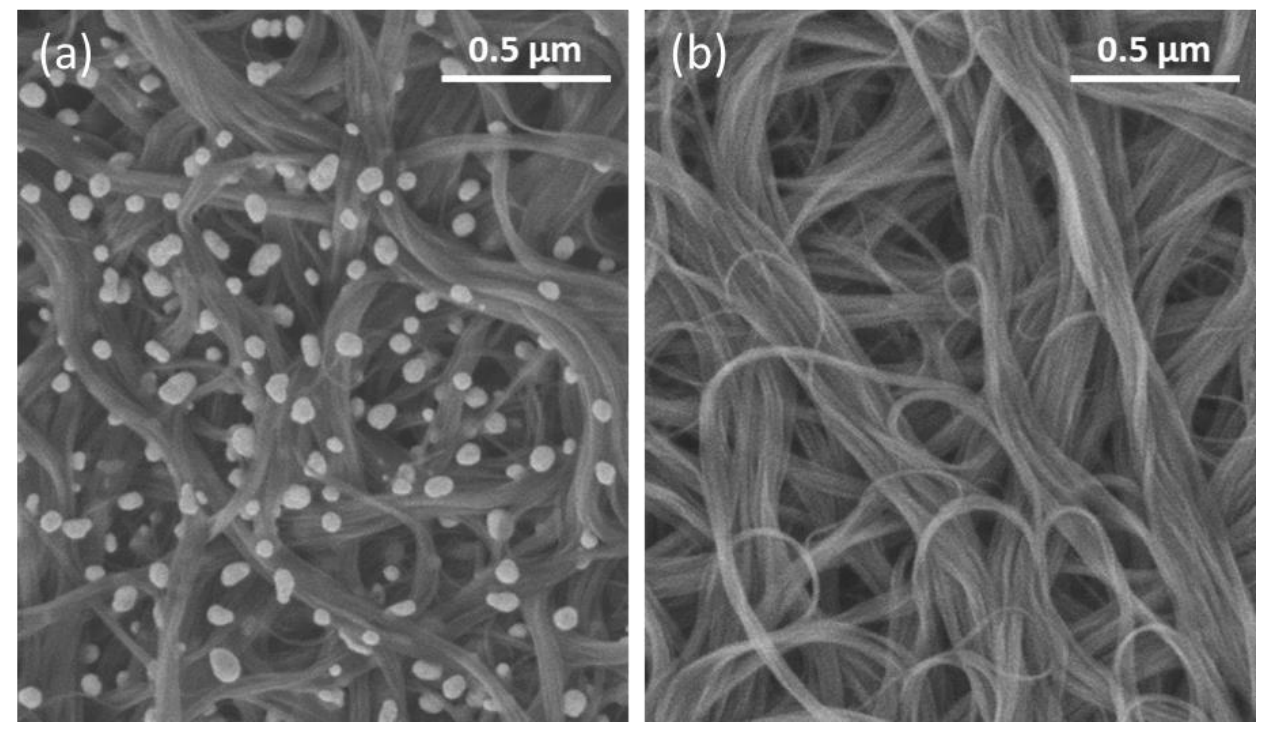

Figure S10. Spatial defect profiling is achieved through electroless gold deposition, and the Au-NP density is characterized by the SEM micrographs of a tagged SWCNT sample after (a) ion irradiation to a fluence of $1 \times 10^{15}$ ions $/ \mathrm{cm}^{2}$ and (b) after subsequent thermal annealing at $1000^{\circ} \mathrm{C}$ in $\mathrm{H}_{2} / \mathrm{Ar}(5 \% / 95 \% \mathrm{~mol} / \mathrm{mol})$ forming gas.

\section{$\underline{\text { References: }}$}

1. Landi, B. J.; Cress, C. D.; Evans, C. M.; Raffaelle, R. P. Thermal Oxidation Profiling of Single-Walled Carbon Nanotubes. Chem Mater, 2005, 17, 6819-6834.

2. Landi, B. J.; Ruf, H. J.; Evans, C. M.; Cress, C. D.; Raffaelle, R. P. Purity Assessment of Single-Wall Carbon Nanotubes, Using Optical Absorption Spectroscopy. J Phys Chem B, 2005, 109, 9952-9965.

3. Dresselhaus, M. S.; Jorio, A.; Saito, R. Characterizing Graphene, Graphite, and Carbon Nanotubes by Raman Spectroscopy. Annu Rev Conden Ma P, 2010, 1, 89-108.

4. Dresselhaus, M. S.; Jorio, A.; Souza, A. G.; Saito, R. Defect Characterization in Graphene and Carbon Nanotubes Using Raman Spectroscopy. Philos TR Soc A, 2010, 368, 5355-5377.

5. Cox, N. D.; Rossi, J. E.; Cress, C. D.; Merrill, A.; Crompton, K. R.; Landi, B. J. Spatially Selective Au Nanoparticle Deposition and Raman Analysis of Ion-Irradiated Single-Wall Carbon Nanotubes. J Phys Chem C, 2014, 118, 14031-14038. 